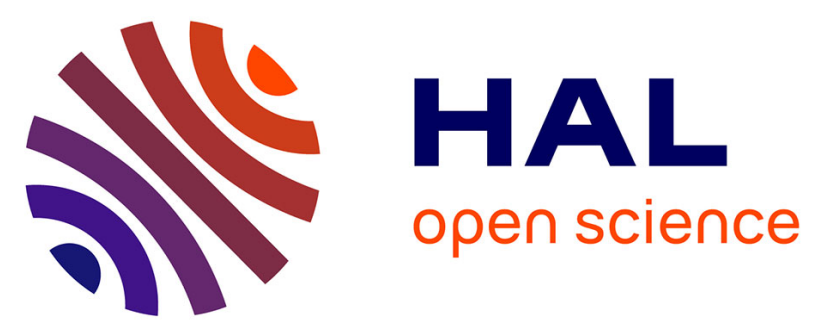

\title{
Prediction of the intramembranous tissue formation during perisprosthetic healing with uncertainties. Part 2. Global clinical healing due to combination of random sources
}

Ji Yang, Béatrice Faverjon, David Dureisseix, Pascal Swider, Steffen Marburg, Herwig Peters, Nicole Kessissoglou

\section{To cite this version:}

Ji Yang, Béatrice Faverjon, David Dureisseix, Pascal Swider, Steffen Marburg, et al.. Prediction of the intramembranous tissue formation during perisprosthetic healing with uncertainties. Part 2. Global clinical healing due to combination of random sources. Computer Methods in Biomechanics and Biomedical Engineering, 2016, 19 (13), pp.1387-1394. 10.1080/10255842.2016.1143465 . hal01345511

\author{
HAL Id: hal-01345511 \\ https://hal.science/hal-01345511
}

Submitted on 15 Nov 2018

HAL is a multi-disciplinary open access archive for the deposit and dissemination of scientific research documents, whether they are published or not. The documents may come from teaching and research institutions in France or abroad, or from public or private research centers.
L'archive ouverte pluridisciplinaire $\mathbf{H A L}$, est destinée au dépôt et à la diffusion de documents scientifiques de niveau recherche, publiés ou non, émanant des établissements d'enseignement et de recherche français ou étrangers, des laboratoires publics ou privés. 


\title{
Prediction of the intramembranous tissue formation during perisprosthetic healing with uncertainties. Part 2. Global clinical healing due to combination of random sources.
}

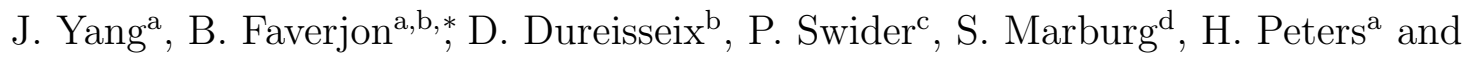 \\ N. Kessissoglou ${ }^{\mathrm{a}}$
}

\begin{abstract}
${ }^{a}$ School of Mechanical and Manufacturing Engineering, UNSW Australia
${ }^{b}$ Université de Lyon, CNRS, INSA-Lyon, LaMCoS UMR5259, France

${ }^{c}$ Université de Toulouse, CNRS, INP-Toulouse, IMFT UMR 5502, France

${ }^{d}$ Institut für Mechanik, Universität der Bundeswehr München, Germany
\end{abstract}

\begin{abstract}
This work proposes to examine the variability of the bone tissue healing process in the early period after the implantation surgery. The first part took into account the effect of variability of individual biochemical and mechanical factors on the solid phase fraction, which is an indicator of the quality of the primary fixation and condition of its long-term behaviour. The next issue, addressed in this second part, is the effect of cumulative sources of uncertainties on the same problem of a canine implant. This paper is concerned with the ability to increase the number of random parameters to assess the coupled influence of those variabilities on the tissue healing. To avoid an excessive increase in the complexity of the numerical modelling and further, to maintain efficiency in computational cost, a collocation-based polynomial chaos expansion approach is implemented. A progressive set of simulations with an increasing number of sources of uncertainty is performed. This information is helpful for future implant design and decision process for the implantation surgical act.

This document is a preprint of the following published article. The Version of Record of this manuscript has been published and is available in Computer Methods in Biomechanics and Biomedical Engineering, 2016

http://www.tandfonline.com/10.1080/10255842.2016.1143465
\end{abstract}

Keywords: Implant Fixation; Stochastic Model; Combined uncertainties; Collocation-based polynomial chaos expansion; Biomechanics

\section{Introduction}

The fixation of an orthopaedic implant to the surrounding bone greatly affects its clinical longevity $[7,19]$. The implant fixation quality is determined by the bone healing process in the early period after the implantation surgery $[13,16]$.

Several numerical models for tissue evolution prediction are available in the literature and were discussed in the first part of this study. In the biomechanical model used herein, the bone tissue was modelled as a multiphasic porous medium and coupled with computational cell biology $[2,1,6]$. Numerical results from the coupled biochemical-mechanical model were validated by experimental results from a canine implant $[17,21]$.

The healing process of a bone implant is affected by significant uncertainties from the mechanical and biochemical environments $[20,12]$. The influence of system uncertainties can be observed directly using Monte Carlo simulations (MCS) [4], which require a large number of simulations and high computational cost to obtain accurate results.

The first part of this study focussed on the influence of individual uncertainties on the healing process. A biomechanical model was combined with an intrusive stochastic method, the Galerkin projection

\footnotetext{
${ }^{*}$ Corresponding author. Email: b.faverjon@unsw.edu.au
} 
polynomial chaos expansion (PCE). Uncertainty propagation during this process was predicted at a low computational cost.

For the bone-implant healing problem considered in this work, coupling in the influence of uncertain parameters requires prediction of the effect of an increasing number of simultaneous sources of variability on the biomechanical phenomena of healing, while keeping again the simulation cost affordable. This is the focus of this second article.

Due to its computational efficiency, the PCE method has been widely applied in many disciplines for uncertainty quantification problems. The computational efficiency of PCE was compared with numerical cubature scheme, Karhunen-Loeve moment equation method and MCS for a groundwater flow with uncertain conductivity in porous medium [15]. It was found that all these methods suffered from the curse of dimensionality as the system size and the number of uncertainty increased and that PCE outperformed other methods when the appropriate truncated number of base polynomials were identified to quantify the uncertainties. For the solution of the PCE coefficients, the PCE method can be divided into intrusive and non-intrusive techniques $[8,14]$. In the intrusive PCE method, the PCE coefficients are solved by the stochastic Galerkin projection [5], which requires access to the system equations and results in more complex system equations. This accurate method was used in the first part of the paper to examine the effect of individual biochemical and mechanical uncertainties on the bone-implant healing process.

In the non-intrusive PCE method, the system equations are treated as a black box and the calculation of PCE coefficients is based on a set of deterministic simulations, which is more amenable in terms of computational cost for large-scale models and in terms of modelling complexity for iterative methods. To calculate the PCE coefficients, two non-intrusive approaches can be used: the spectral projection method and the collocation-based method [3]. The spectral projection method projects the output results into the base polynomials using an orthogonality property and multidimensional integral, which involves random sampling, quadrature, Strouds cubature formula [18], or sparse grid approaches [22]. The collocation-based method uses a linear regression algorithm that approximates the PCE coefficients to match the output results from the deterministic model at a set of collocation points using the least square algorithm [8], which is more straightforward to implement than the spectral projection. The collocation-based PCE method was combined with Karhunen-Loeve expansion to analyse the flow in porous media with an uncertain hydraulic conductivity field [11]. This method was applied to estimate internal and biologically effective doses of toxic chemicals for the human body and to predict the pollutant concentrations in the atmosphere [9], and showed higher computational efficiency compared with standard and modified MC simulations. To examine the forces and moments of intervertebral discs in the human spine, the collocation-based PCE was shown to be more accurate than the spectral projection method [10].

This paper investigates the combined effects of random biochemical and mechanical parameters on the bone-implant healing process using collocation-based polynomial chaos expansion. The biomechanical model considers both mechanical and biochemical equations to take into account the osteoblast cells migration, growth factors diffusion and bone deposit. Since the collocation-based method introduces additional approximations (least-square at the collocation points), its validation is performed with comparison to MCS in terms of accuracy and computational cost. Its performances are exemplified on the case of a canine implant, and insight on the healing process with respect to the sources of uncertainty are discussed.

\section{A biomechanical model with combined random factors}

\subsection{Deterministic model as a black box}

The healing process with tissue formation is modeled as a transient convective-diffusive-reactive problem in porous media [2]. Its parabolic nature allows derivation of finite difference approximation schemes, see [23] and the first part of this paper, in terms of a 4-field problem involving the bone solid fraction $\phi^{s}$, the concentration of osteoblast cells $C^{c}$, the concentration of growth factors $C^{M}$, and the relative fluid flow rate $-\boldsymbol{q}^{f}$ (Darcy velocity). The formal expression of the evolution problem requires $x=$ $f\left(x, \operatorname{div} \operatorname{grad} x, \boldsymbol{q}^{f}\right)$ where the state vector is $x=\left[\begin{array}{lll}\phi^{s} & \left(1-\phi^{s}\right) C^{c} & \left(1-\phi^{s}\right) C^{M}\end{array}\right]^{T}$, closed with a fluid conservation equation in the case of incompressibility, that reads $-\phi^{s}=\operatorname{div} \boldsymbol{q}^{f}$. The function $f$ is not detailed herein and the interested reader is suggested to refer to [2]. Despite the macroscopic nature of the model, the coupling of the four fields in function $f$ induces a potentially large number of phenomenological parameters. The main uncertainties have been identified as the coefficient of osteoid synthesis $\alpha^{s}$, the coefficient of haptotactic migration $h^{c}$, the coefficient of chemotactic migration $\chi^{c}$, 
and the drill hole radius $r_{d}[2]$. The ranges of these uncertain parameters are listed in Table 1 . In the following analyses, these parameters are assumed to follow uniform distributions. Depending on the study case, some inputs will be considered as random and some others as deterministic whose values are chosen by the measurements given in [2].

Table 1: Parameters that may encounter variability

\begin{tabular}{cc}
\hline Parameter & Range \\
\hline$\alpha^{s} / \mathrm{mm}^{6} \cdot \mathrm{cell}^{-1} \cdot \mathrm{ng}^{-1} \cdot \mathrm{s}^{-1}$ & {$[1,5] \times 10^{-9}$} \\
$h^{c} / \mathrm{mm}^{5} \cdot \mathrm{s}^{-1} \cdot \mathrm{kg}^{-1}$ & {$[0.04,0.8]$} \\
$\chi^{c} / \mathrm{mm}^{5} \cdot \mathrm{s}^{-1} \cdot \mathrm{ng}^{-1}$ & {$[1,14.5] \times 10^{-5}$} \\
$r_{d} / \mathrm{mm}$ & {$[3.8,4.4]$} \\
\hline
\end{tabular}

Using a stochastic analysis, these uncertain parameters are considered as inputs of the model. The output quantity of interest is the bone solid fraction field $\phi^{s}$ once the transient regime is stabilized (after 56 days). For the current application of a canine implant, recalled in part 1 of the paper, a 1D axisymmetric spatial discretization is used, so that the quantity of interest is $\phi^{s}(r)$.

In this first part of the paper, the input parameters and output solutions were considered as random and represented by a polynomial chaos expansion (PCE). Using an explicit finite difference scheme and Galerkin projection, the stochastic system equations are transformed to a set of equations to solve the deterministic PCE coefficients for the output results, leading to an intrusive approach. This approach is amenable for the stochastic analysis of each input separately (in each case a single input is considered as uncertain, while the other ones take their nominal deterministic values). As such, the individual uncertainty analysis in part 1 is equivalent to a stochastic sensitivity analysis for each parameter.

It is now of interest to quantify the effect of coupled uncertainties, to provide further insight into the healing process and to be used as a tool during the decision process of the implantation surgical act. The complexity of the previous intrusive approach increases with the number of uncertain parameters taken into account. It is therefore not suited for the coupled analysis. A non-intrusive approach is now required to address the aforementioned issues that will allow the solver to be considered as a black box, and the same former deterministic model to be re-used as in a Monte Carlo approach.

\subsection{Non-intrusive polynomial chaos expansion}

The stochastic method used here is the stochastic response surface method using collocation-based PCE [8]. The output is expanded in a polynomial chaos expansion by [5]

$$
\phi^{s}(r, \boldsymbol{\xi}) \approx \sum_{i=0}^{N_{\phi}} \phi_{i}^{s}(r) \Psi_{i}(\boldsymbol{\xi})
$$

in which the number of unknown polynomial coefficients is equal to $N_{\phi}+1=(n+p) ! / n ! / p$ ! with $p$ the PCE order and $n$ the number of random variables of inputs $\boldsymbol{\xi}=\left(\xi_{1}, \xi_{2} \ldots \xi_{n}\right)$. The random inputs follow the uniform probability law and the base polynomials $\Psi_{i}$ defined in equation (1) are mutually orthogonal Legendre polynomials. The collocation-based method outputs are calculated at a set of collocation points $\left(\boldsymbol{\xi}_{0}, \boldsymbol{\xi}_{1} \ldots \boldsymbol{\xi}_{N_{\xi}}\right)$ in the parameter space from the deterministic model. The number of collocation points should be greater than the number of unknown PCE coefficients, and they are chosen as the roots of a higher $p+1$ order polynomial to capture the points from the region of high probability. The unknown PCE coefficients are then determined as the least square solution arising from the minimization of the norm of the residual in equation (1):

$$
\min _{\phi_{i}^{s}(r)} \sum_{k=0}^{N_{\xi}}\left[\phi^{s}\left(r, \boldsymbol{\xi}_{k}\right)-\sum_{i=0}^{N_{\phi}} \phi_{i}^{s}(r) \Psi_{i}\left(\boldsymbol{\xi}_{k}\right)\right]^{2}
$$

to solve a linear system of equations.

\section{Numerical results}

Two typical healing patterns encountered in the animal models (herein, a canine experiment) were selected to support the computational developments. They were classified according to the amount of 
solid fraction distribution $\phi^{s}$ and designated as good healing $(\mathrm{GH})$ when the average solid fraction was in the range of that of the host bone and poor healing $(\mathrm{PH})$ for significant lower values. Ex-vivo histological data from [2] were included and compared with the numerical results obtained from the present model.

It was shown previously in the first part of the paper that the coefficient of osteoid synthesis $\alpha^{s}$ had an impact at both the implant surface $r_{i}$ and drill hole $r_{d}$ for GH, but only at the drill hole radius $r_{d}$ for $\mathrm{PH}$. In comparison, the haptotactic coefficient $h^{c}$ showed less effect even if it influenced the homogeneity of the solid fraction into the post-operative gap, especially for GH. The chemotactic coefficient $\chi^{c}$ played a significant role in tissue formation with a peak at the implant radius $r_{i}$ for both $\mathrm{GH}$ and $\mathrm{PH}$. For $\mathrm{PH}$, the experimental results were observed to be close to the lower limit of the PCE envelope. Variations of the drill hole radius $r_{d}$ had a significant impact on the tissue formation at the drill hole and it modified the homogeneity of neo-formed tissue in the gap $r_{i}-r_{d}$, especially for GH.

Combined uncertainties in the various relevant biochemical factors and the drill hole radius on the implant healing process are now examined. The selected combinations were those that brought (i) a good prediction of experimental data and (ii) relevant clues to progress in the interpretation of clinical results. Each input follows a uniform distribution within the range shown in Table 1 [2], and are identical to the values in part 1 of the paper. The input parameters are well represented by the 1st order Legendre PCE. In all cases, converged stochastic numerical results are obtained using polynomial chaos expansion of third order $(p=3)$, corresponding to 16 collocation points for two uncertain parameters and 64 collocation points for three uncertain parameters in equation (2). Compared with the 50000 Monte Carlo computations of the deterministic model, the collocation-based PCE computations provide significantly reduced computational cost with an equivalent accuracy. For each case of uncertainty, results are presented in terms of the mean and variance of the solid fraction distribution $\phi^{s}$. Upper and lower envelopes of $\phi^{s}$ are constructed by taking the maximum and minimum values of 50000 Legendre polynomial samples $\boldsymbol{\xi}$ for the solid fraction.

\subsection{Combined uncertainties involving two parameters}

\subsubsection{Active migration: haptotactic and chemotactic coefficients $h^{c}$ and $\chi^{c}$}

Figure 1 showed the combined influences of active migration parameters $h^{c}$ and $\chi^{c}$. Comparison with the part 1 of the paper for individual parameters confirmed the major role played by $\chi^{c}$ even if a smoother variance evolution provided by $h^{c}$ was detected into the post-operative gap $\left(r_{i}, r_{d}\right)$.

\subsubsection{Healing capability and surgical technique: coefficient of osteoid synthesis $\alpha^{s}$ and drill hole radius $r_{d}$}

Figure 2 shows average trends when using combined uncertainties $\alpha^{s}$ and $r_{d}$ that are similar to those that can be obtained with individual uncertainties (see Part 1 of this paper) while their variances are more accentuated.

\subsection{Combined uncertainties involving three parameters}

The combinations of three parameters were then investigated. The role of active migrations $\left(\chi^{c}, h^{c}\right)$ when associated with uncertainties in bone tissue formation $\left(\alpha^{s}\right)$ or surgical technique $\left(r_{d}\right)$ are examined as follows.

\subsubsection{Osteoid synthesis and active migrations: $\alpha^{s}, \chi^{c}$ and $h^{c}$}

The gap region between the implant and drill hole $\left(r \in\left[r_{i}, r_{d}\right]\right)$ is still observed to be the location of significant disturbances as shown in Figure 3. The coefficient of osteoid synthesis $\alpha^{s}$ adds more disturbances to the host bone $\left(r \in\left[r_{d}, r_{s}\right]\right.$, where $r_{s}$ is the limit of the region of influence for the healing process) in terms of mean values and variance of solid fraction $\phi^{s}$. Compared with previous results for combined uncertainty in the haptotactic and chemotactic migrations $\left(\chi^{c}, h^{c}\right)$, Figure 1 does not show strong differences in terms of shape for the radial evolution of $\phi^{s}$ when uncertainty in the osteoid synthesis is included.

\subsubsection{Active migrations and surgical technique: $r_{d}, \chi^{c}$ and $h^{c}$}

Compared to results shown in Figure 1, the combination of active migrations with the uncertainties on the drill hole (Figure 4) did not induce fundamental differences in the tissue healing distribution pattern. 

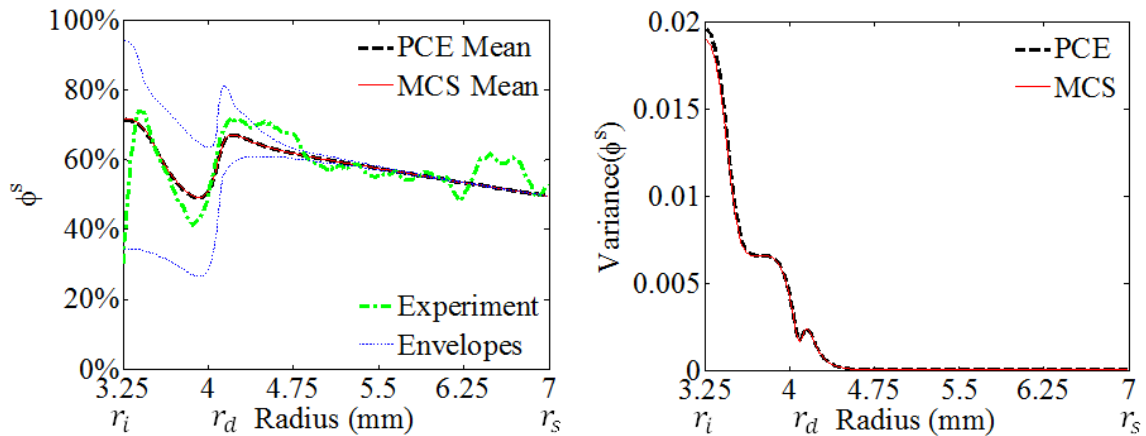

(a) Good healing.
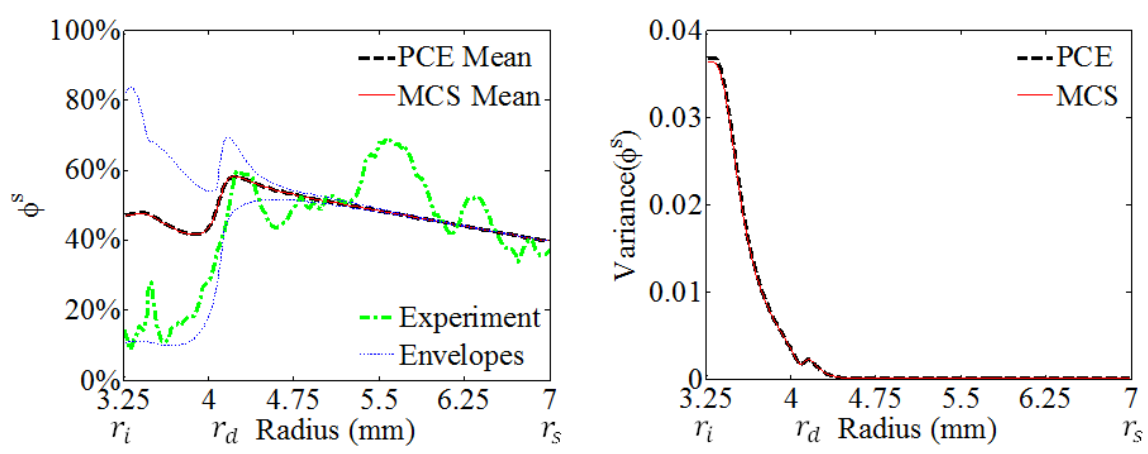

(b) Poor healing.

Figure 1: Influence of uncertain coefficients of haptotactic and chemotactic migrations $h^{c}$ and $\chi^{c}$.
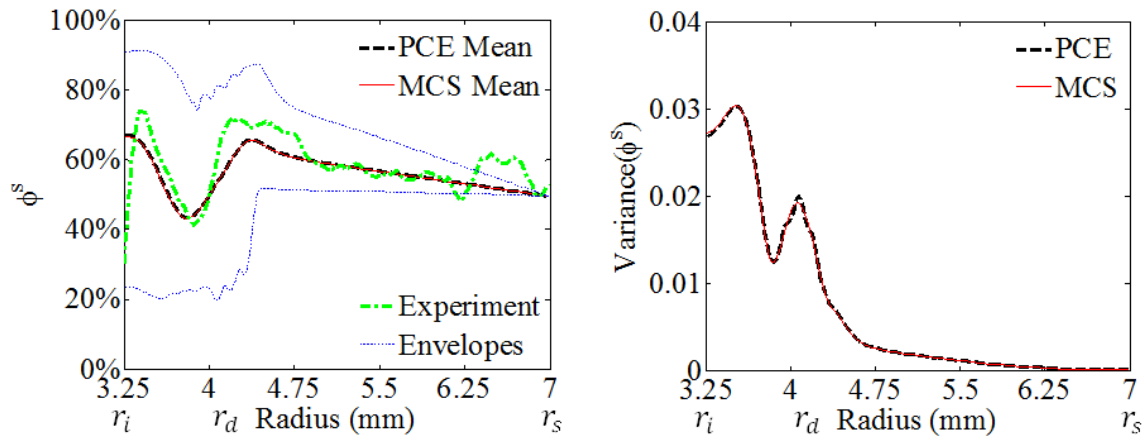

(a) Good healing.
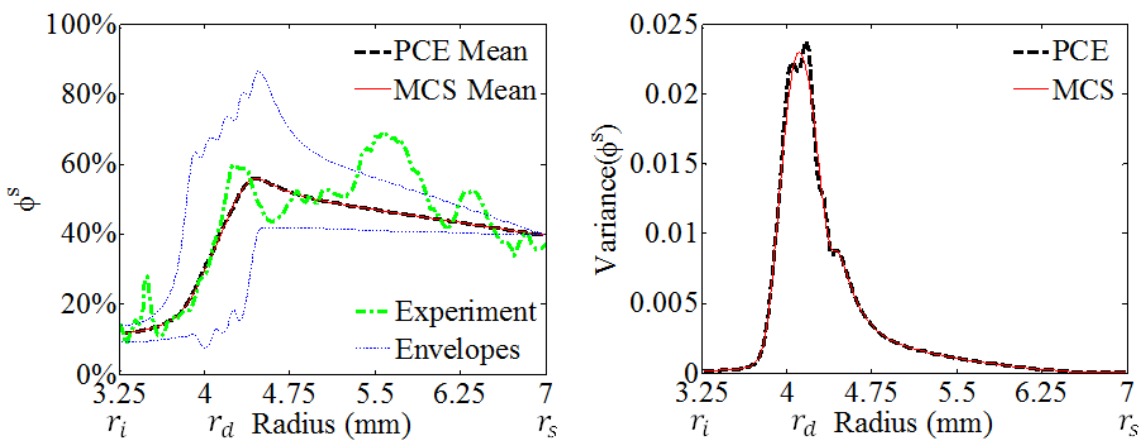

(b) Poor healing.

Figure 2: Influence of uncertain coefficients of osteoid synthesis $\alpha^{s}$ and drill hole radius $r_{d}$. 

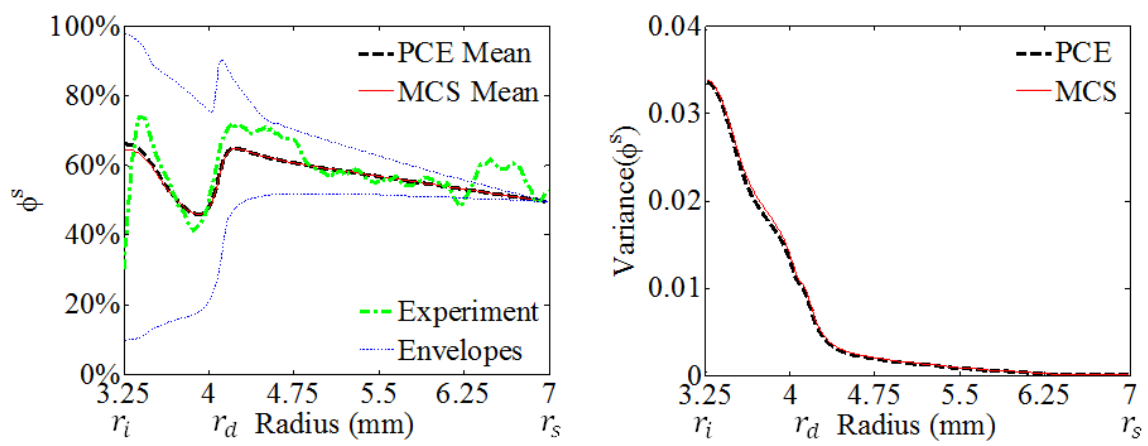

(a) Good healing.
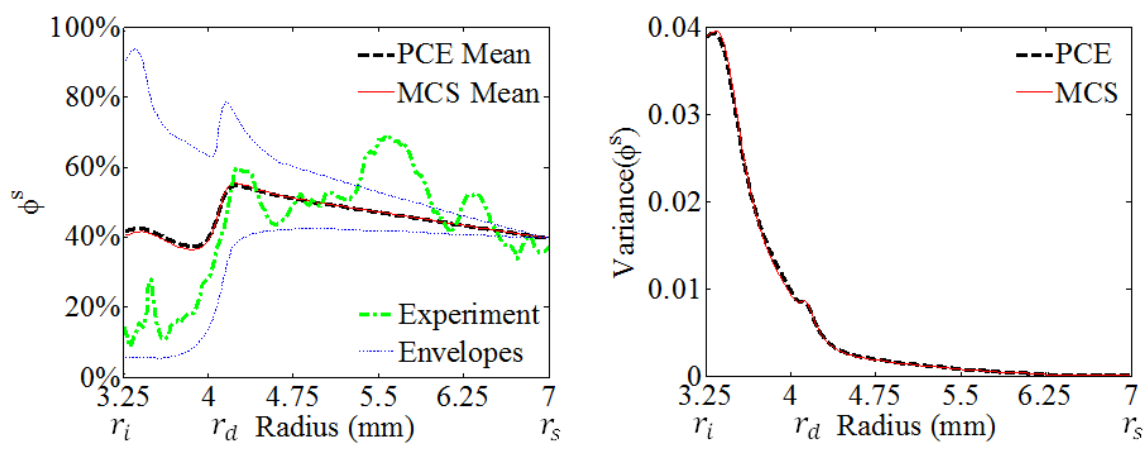

(b) Poor healing.

Figure 3: Influence of uncertain coefficients of osteoid synthesis $\alpha^{s}$, haptotactic migration $h^{c}$ and chemotactic migration $\chi^{c}$.

The obtained variance smoothing was associated with a small increase of the solid fraction envelope size.

\subsubsection{Osteoid synthesis, active migration and surgical technique: $\alpha^{s}, \chi^{c}$ and $r_{d}$}

When the combination of $\left(\alpha^{s}, r_{d}\right)$ described by the responses in Figure 2 was associated with uncertainties in $\chi^{c}$, the mean values and envelope results are significantly modified in magnitude, and particularly in shape for the PH case. As shown in Figure 5, maximal variances were obtained and the heterogeneity of the solid fraction variance was intensified in the full region of interest $\left(r_{i}, r_{d}\right)$. Finally, the envelope of solutions in Figure 5(b) was able to encompass both cases $\mathrm{GH}$ and $\mathrm{PH}$.

\section{Discussion and Conclusions}

Clinically, the main issue is the primary fixation and consistent healing between the surface of the implant and the host tissue is generally a good indicator for long-term survival of the arthroplasty. The amount of structural (or mineralized) fraction into the neo-formed tissue is the result of combined and complex biomechanical events. The influence of the variability of the various parameters is therefore significant and not trivial to estimate.

The numerical methodology proposed in this work can be used to examine the effects of biomechanical factors in the periprosthetic healing. Results obtained by using collocation-based polynomial chaos expansion (PCE) were in excellent agreement with Monte Carlo simulations (MCS) and offered a drastic reduction of computational time. Furthermore, PCE can predict the mean value, envelopes and variance. Small differences between the PCE and MCS results were attributed to the nonlinear uncertainty from the drill hole radius $r_{d}$, which is independent of the PCE order and corresponds to a limitation of collocation-based PCE [9]. These small discrepancies do not appear in the use of the intrusive PCE method described in part 1. However the intrusive method based on Galerkin projection PCE is more computationally expensive than the collocation-based PCE method for the case of several random inputs.

Homogeneity of the healing process was conditioned by haptotaxis migration that emphasized adhesion gradients at the drill hole in opposition to chemotaxis migration conditioned by the attraction of 

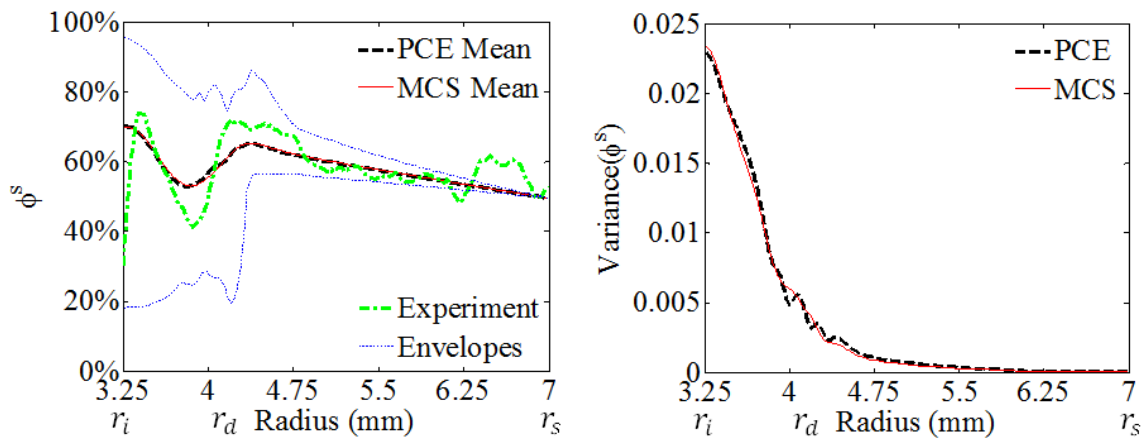

(a) Good healing.
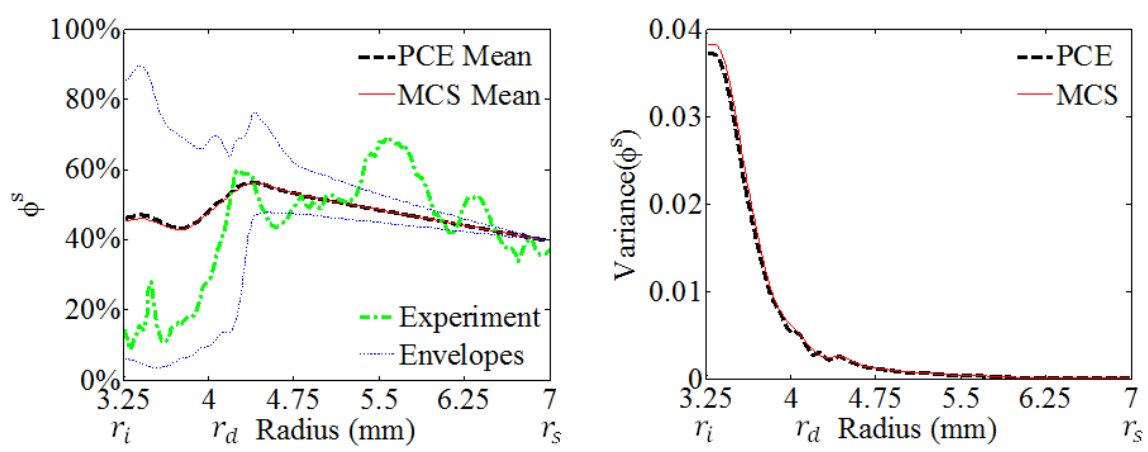

(b) Poor healing.

Figure 4: Influence of uncertain coefficients of drill hole radius $r_{d}$, chemotactic and haptotactic migrations $h^{c}$ and $\chi^{c}$.
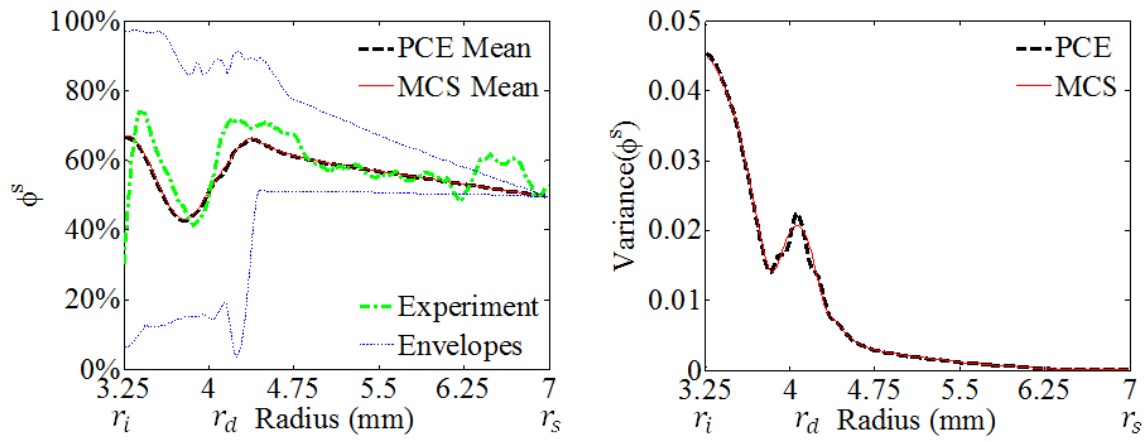

(a) Good healing
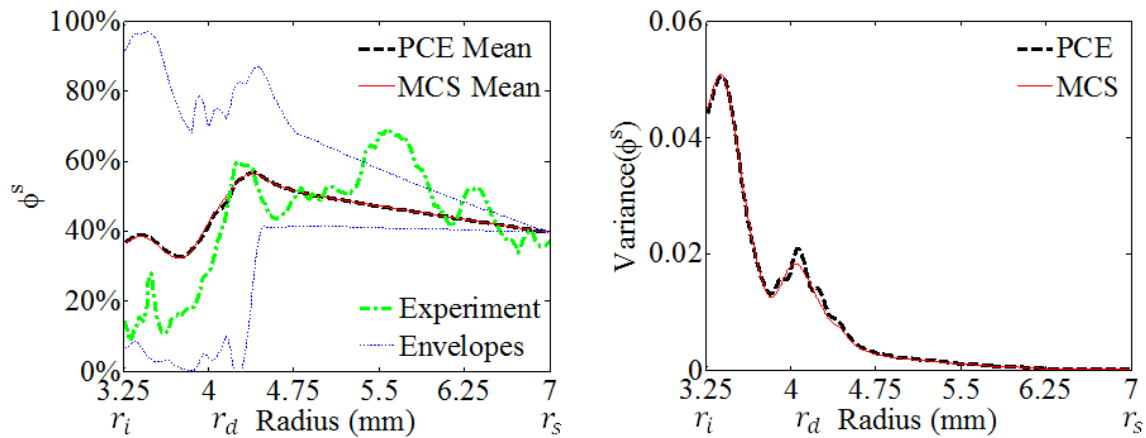

(b) Poor healing.

Figure 5: Influence of uncertain coefficients of osteoid synthesis $\alpha^{s}$, chemotactic migration $\chi^{c}$ and drill hole radius $r_{d}$. 
growth factors on the implant surface. These effects were well corroborated by the combined uncertainty analyses that highlight the leading role of chemo attractants.

When uncertainty of the drill-hole radius was combined with that of osteoid synthesis, the numerical model confirmed that the drill-hole zone was the site of significant effects and to a lesser extent, the implant surface was affected.

The numerical methodology allowed triple uncertainties to be evaluated simultaneously and revealed that the combination of active migrations to osteoid synthesis or drill-hole radius did not provide more significant information excepting the increase of variances.

The combination of chemotactic migration with osteoid synthesis and drill-hole radius was shown to play a major role in variation in the healing process. This combination was able to encompass the healing patterns previously defined $(\mathrm{GH}, \mathrm{PH})$ in a unified approach. In a clinical setting, the envelope of solutions in Figure 5(b) was conditioned by the surgical technique $\left(r_{d}\right.$ parameter) which influenced the primary fixation and the biochemical potential of the site (blood clot, autologous growth factors, pre-osteoblasts population), and the role of implant bioactive coating.

The approach adopted here applied to the case of canine implant provides insights on the healing implant for several sources of uncertainty. The objective quantification of mechanobiological events and the prediction of their variability contributed to a better understanding of the source of diversity observed in-vivo. This information is helpful for future implant design and decision process for the implantation surgical act.

\section{Acknowledgments}

This work was completed thanks to J. E. Bechtold PhD (Departments of Orthopaedic Surgery, Mechanical and Biomedical Engineering, University of Minnesota USA) and K. Søballe MD, PhD (University Hospital of Aarhus, Denmark). Experimental studies were conducted with the support of NIH USA (AR 42051). B. Faverjon gratefully acknowledges the French Education Ministry, University of Lyon, CNRS, INSA of Lyon and LabEx iMUST for the CRCT and the out mobility grant.

\section{References}

[1] D. Ambard, G. Guérin, and P. Swider. A reactive poroelastic model to predict the periprosthetic tissue healing. European Journal of Computational Mechanics, 18(1):131-143, 2009.

[2] D. Ambard and P. Swider. A predictive mechano-biological model of the bone-implant healing. European Journal of Mechanics A/Solids, 25:927-937, 2006.

[3] M. S. Eldred. Recent advances in non-intrusive polynomial chaos and stochastic collocation methods for uncertainty analysis and design. In 50th AIAA/ASME/ASCE/AHS/ASC Structures, Structural Dynamics, and Materials Conference, Palm Springs, 2009.

[4] G. S. Fishman. Monte Carlo: concepts, algorithms, and applications. Springer series in operations research. Springer, New York, Berlin, 1995.

[5] R. G. Ghanem and P. D. Spanos. Stochastic Finite Elements: A Spectral Approach. Springer-Verlag, New York, 1991.

[6] G. Guérin, D. Ambard, and P. Swider. Cells, growth factors and bioactive surface properties in a mechanobiological model of implant healing. Journal of Biomechanics, 42(15):2555 - 2561, 2009.

[7] M. Hahn, M. Vogel, F. Eckstein, M. Pompesius-Kempa, and G. Delling. Bone structure changes in hip joint endoprosthesis implantation over the course of many years. A quantitative study. Der Chirurg., 59(11):782-787, 1988. In German.

[8] S. Huang, S. Mahadevan, and R. Rebba. Collocation-based stochastic finite element analysis for random field problems. Probabilistic Engineering Mechanics, 22(2):194 - 205, 2007.

[9] S. S. Isukapalli, A. Roy, and P. G. Georgopoulos. Stochastic response surface methods (SRSMs) for uncertainty propagation: Application to environmental and biological systems. Risk Analysis, 18(3):351-363, 1998. 
[10] N. Karajan, D. Otto, S. Oladyshkin, and W. Ehlers. Application of the polynomial chaos expansion to approximate the homogenised response of the intervertebral disc. Biomechanics and Modeling in Mechanobiology, 13(5):1065-1080, 2014.

[11] H. Li and D. Zhang. Probabilistic collocation method for flow in porous media: Comparisons with other stochastic methods. Water Resources Research, 43(9):W09409, 2007.

[12] S. Miramini, L. Zhang, M. Richardson, M. Pirpiris, P. Mendis, K. Oloyede, and G. Edwards. Computational simulation of the early stage of bone healing under different configurations of locking compression plates. Computer Methods in Biomechanics and Biomedical Engineering, 18(8):900$913,2015$.

[13] S. Morshed, K. J. Bozic, M. Ries, H. Malchau, and J. J. Colford. Comparison of cemented and uncemented fixation in total hip replacement: a meta-analysis. Acta Orthop., 78(3):315-26, 2007.

[14] S. Oladyshkin and W. Nowak. Data-driven uncertainty quantification using the arbitrary polynomial chaos expansion. Reliability Engineering 8 System Safety, 106:179 -190, 2012.

[15] C. Rupert and C. Miller. An analysis of polynomial chaos approximations for modeling singlefluid-phase flow in porous medium systems. Journal of Computational Physics, 226(2):2175 - 2205, 2007.

[16] F. Schwarz, M. Herten, M. Sager, M. Wieland, M. Dard, and J. Becker. Histological and immunohistochemical analysis of initial and early osseous integration at chemically modified and conventional sla titanium implants: preliminary results of a pilot study in dogs. Clinical Oral Implants Research, 18(4):481-488, 2007.

[17] K. Søballe, E. S. Hansen, H. B-Rasmussen, P. H. Jørgensen, and C. Bünger. Tissue ingrowth into titanium and hydroxyapatite-coated implants during stable and unstable mechanical conditions. Journal of Orthopaedic Research, 10(2):285-299, 1992.

[18] A. H. Stroud. Remarks on the disposition of points in numerical integration formulas. Mathematics of Computation, 11:257-261, 1957.

[19] P. Swider, D. Ambard, G. Guérin, K. Søballe, and J. E. Bechtold. Sensitivity analysis of periprosthetic healing to cell migration, growth factor and post-operative gap using a mechanobiological model. Computer Methods in Biomechanics and Biomedical Engineering, 14(9):763-771, 2011.

[20] R. Vayron, E. Barthel, V. Mathieu, E. Soffer, F. Anagnostou, and G. Haiat. Variation of biomechanical properties of newly formed bone tissue determined by nanoindentation as a function of healing time. Computer Methods in Biomechanics and Biomedical Engineering, 14(sup1):139-140, 2011.

[21] M. T. Vestermark, J. E. Bechtold, P. Swider, and K. Søballe. Mechanical interface conditions affect morphology and cellular activity of sclerotic bone rims forming around experimental loaded implants. Journal of Orthopaedic Research, 22(3):647-652, 2004.

[22] D. Xiu and J. S. Hesthaven. High-order collocation methods for differential equations with random inputs. SIAM Journal on Scientific Computing, 27(3):1118-1139, 2005.

[23] J. Yang, B. Faverjon, D. Dureisseix, P. Swider, and N. Kessissoglou. Analysis of bone-implant healing process with uncertain properties using polynomial chaos expansion. In 43rd International Congress on Noise Control Engineering - inter.noise 2014, Melbourne, Australia, november 2014. 